in the moist substance is to keep the bases of the icy filaments, or the lower side of the stratum formed by their agglutination, wet; and congelation of this film appears to be continuous.

$$
\text { W. J. MCGEE }
$$

U.S. Geological Survey, Washington, D.C., U.S.A., February I

\section{Four-Dimensional Space}

Possibly the question, What is the fourth dimension? may admit of an indefinite number of answers. I prefer, therefore, in proposing to consider Time as a fourth dimension of our existence, to speak of it as $a$ fourth dimension rather than the fourth dimension. Since this fourth dimension cannot be introduced into space, as commonly understood, we require a new kind of space for its existence, which we may call time-spacé. There is then no difficulty in conceiving the analogues in this new kind of space, of the things in ordinary space which are known as lines, areas, and solids. A straight line, by moving in any direction not in its own length, generates an area; if this area moves in any direction not in its own plane it generates a solid; but if this solid moves in any direction, it still generates a solid, and nothing more. The reason of this is that we have not supposed it to move in the fourth dimension. If the straight line moves in its own direction, it describes only a straight line ; if the area moves in its own plane, it describes only an area; in each case, motion in the dimensions in which the thing exists, gives us only a thing of the same dimensions; and, in order to get a thing of higher dimensions, we must have motion in a new dimension. But, as the idea of motion is only applicable in space of three dimensions, we must replace it by another which is applicable in our fourth dimension of time. Such an idea is that of successive existence. We must, therefore, conceive that there is a new three-dimensional space for each successive instant of time ; and, by picturing to ourselves the aggregate formed by the successive positions in time-space of a given solid during a given time, we shall get the idea of a four-dimensional solid, which may be called a sur-solid. It will assist us to get a clearer idea, if we consider a solid which is in a constant state of change, both of magnitude and position; and an example of a solid which satisfies this condition sufficiently well, is afforded by the body of each of us. Let any man picture to himself the aggregate of his own bodily forms from birth to the present time, and he will have a clear idea of a sur-solid in time-space.

Let us now consider the sur-solid formed by the movement, or rather, the successive existence, of a cube in time-space. We are to conceive of the cube, and the whole of the three-dimensional space in which it is situated, as floating away in time-space for a given time; the cube will then have an initial and a final position, and these will be the end boundaries of the sur-solid. It will therefore have sixteen points, namely, the eight points belonging to the initial cube, and the eight belonging to the final cube. The successive positions (in time-space) of each of the eight points of the cube, will form what may be called a time-line; and adding to these the twenty-four edges of the initial and final cubes, we see that the sur-solid has thirty-two lines. The successive positions (in time-space) of each of the twelve edges of the cube, will form what may be called a time area; and, adding these to the twelve faces of the initial and final cubes, we see that the sur-solid has twenty-four areas. Lastly, the successive positions (in time-space) of each of the six faces of the cube, will form what may be called a time-solid; and, adding these to the initial and final cubes, we see that the sur-solid is bounded by eight solids. These results agree with the statements in your article. But it is not permissible to speak of the sur-solid as resting in "space," we must rather say that the section of it by any time is a cube resting (or moving) in "space."

March 16

\section{The Action of Very Minute Particles on Light}

THE action upon transmitted light of very minute particles suspended in a transparent medium is very well known, thanks to the investigations of Brücke, Tyndall, and others, up to a certain point. That is to say, that white light, passing through varying depths of a medium with such particles more or less thickly interspersed, is known to emerge coloured yellow, orange, or red, according to the cxtent of the action in question. Wishing to illustrate this phenomenon experimentally, I em- ployed a very dilute solution of sodium thiosulphate (hyposul phite), which was acidified with hydrochloric or sulphuric acid, and then allowed to stand, observing from time to time the appearances when examined by transmitted light. The solution mentioned is admirably adapted for the purpose, inasmuch as the precipitation of the sulphur proceeds gradually; and, according to the greater or less dilution at starting, the completion of the reaction can be spread over a long period of time, in some of my experiments occupying more than forty-eight hours. For a while no turbidity whatever is visible; then a faint opalescence makesits appearance, and these exceedingly minute particles grow gradually in size, remaining, however, quite uniformly suspended for a considerable period, until a dimension is reached which causes them to settle out of the liquid. In this way I observed with unfailing regularity, and in unvarying order, though with various degrees of rapidity, an extension of the series of colours, which, so far as I am aware, had not previously been noticed, or at any rate published. From orange, the tint passed successively through rose red, purplish rose, to a full purple; then by insensible gradations to a fine violet, blue, green, greenish yellow, neutral tint, \&c.

The solution was contained in spherical or pear-shaped flasks, or in cells with flat and parallel sides. A solution which was strong enough to give well-marked yellow, orange, and red tints, was not well adapted for the subsequent stages, as it soon became white and opaque, so that the later colours were almost entirely masked. A half litre flask filled with a solution so dilute, that ten minutes or more elapsed after acidifying before opalescence was first visible, gave very feeble yellow and orange; the rose and rose-purple, though decidedly weak, reminded me in tint of the colours seen towards the upper margin of the recent sky-glows; but when the full purple, violet, and blue were reached, the colours were very strong and well marked. A gas or candle-flame, viewed through the solution, which was violet by transmitted daylight, appeared emerald green. After passing the blue stage, the colours through green and yellow were much weaker, until, as before mentioned, a neutral tint was reached. Beyond this, with such a dilution, nothing further could be satisfactorily observed; but by taking a much more capaciou; flask, and using a solution only one-half or one-third the former strength, faint orange and pink were again observed after passing the neutral point. And with these more dilute solutions, very strongly marked secondary effects were noticed after once passing the "blue stage." A distorted image of a window was formed in the flask, and while the bright portions appeared greenish, those parts where the dark bars of the framework fell, appeared of a fine crimson colour; after the neutral point had been passed, and the bright parts appeared pink, the dark portion of the image appeared a brilliant emerald green. In either of these stages a part of the solution trans. formed to a tall, but narrow glass cylinder, had not sufficient depth to show any perceptible colour when viewed by transmitted light, but placed on a dark background below a window, showed a crimson or green glow respectively when viewed at a certain angle, and a complementary glow when seen at a different angle (by raising or lowering the level of the eye, the cylinder remaining stationary).

With the solution in any given stage of development, the effect of increasing the depth of the column through which the light passed was to increase the saturation of the colour to a large extent, and to alter its tint (apparently in the direction of the less refrangible end of the spectrum) to a much smaller degree. That the colour observed at any given stage was owing mainly to the size of the individual particles rather than to their greater or less proximity, was shown by the fact that, on pouring away half or two-thirds of the contents of the vessel, and filling with water, the colour, although much thinner, was nearly of the same tint.

I am not able to give the proportion by weight of the salt in the solutions experimented with; but I think about one gramme or less to the litre will be found to give good results. One or two trials, however, would soon indicate the appropriate strength.

The character of the colours and the whole nature of the phenomena led me to infer that they were in all probability caused by the interference of light; but as I could not see my way to a rationale of the mode of action, I deferred publication in the hope that hy further investigation their exact nature and true cause might be more clearly worked out. The description in last week's NATURE (p. 439) of Prof. Kiessling's ingenious 